\title{
Local coefficients and the Herbert Formula
}

\author{
Petr M. Akhmet'ev*and Theodore Yu. Popelenskii ${ }^{\dagger}$
}

\section{Introduction}

The Herbert Theorem is used in papers on immersions theory. Let us recall ths theorem. Consider smooth closed manifold $N$ of dimension $n-k$ and consider its immersion $g: N^{n-k} \rightarrow \mathbb{R}^{n}$, which is self-transversal. Then for a manifold

$$
\bar{L}=\{(x, y) \in N \times N \mid x \neq y, g(x)=g(y)\},
$$

which is called a self-intersection manifold of the immersion $g$, the composition $j: \bar{L} \subset N \times N \stackrel{p r_{1}}{\rightarrow} N$ is an immersion. Consider the normal bundle $\nu(g)$ of the immersion $g$. Let us assume that $N$ is oriented. The bundle $\nu(g)$ and the manifold $\bar{L}$ is naturally oriented. The homology class, dual in $N$ to the Euler class of the normal bundle $\nu(g)$, is called the homology Euler class and is denoted by $e_{*}(\nu(g))$. Then in the group $H_{n-2 k}(N, \mathbb{Z})$ the following formula is satisfied (the Herbert formula):

$$
j_{*}[\bar{L}]+e_{*}(\nu(g))=0 .
$$

In the case the manifold $N$ is non-oriented, the formula (1) is satisfied, if homology and cohomology groups is defined over $\mathbb{Z} / 2$-coefficients.

Let us note that the original Herbert's paper [1] contains formulas of $k$-uple self-intersection points of immersions. Here are several generalizations of the Herbert formula, and several approaches to prove it, see [2, 3, 8]. Also self-intersections of immersions are investigated using homology of classifying loop-spaces, see [7].

In the present paper an interest to immersions is related with the classical paper [9], and with relationships to homotopy groups of spheres and of Thom spaces, see [10, 12. Let us mention papers [4, 6, 5] by one of the two authors.

In this paper we discuss a generalisation of the Herbert formula for double points, when the normal bundle of the immersion $g$ admits an additional structure, and an application.

Below the Whitney sum of $k$ copies of a prescribed vector-bundle $\xi$ is denoted by $k \xi$. The trivial line (1-dimensional real) bundle over $N$ is denoted by $\varepsilon_{N}$.

\section{Immersions with additional structure of normal bundles}

Let us define a number of regular immersions cobordism groups. Assume a group $G$ and its representation in the space $\mathbb{R}^{d}$ are fixed. The following vector bundle $\gamma=E G \times{ }_{G} \mathbb{R}^{d} \rightarrow B G$ is well-defined.

Definition 1. Let us say an immersion $g: N^{n-k d} \rightarrow \mathbb{R}^{n}$ is equipped by a G-structure (of its normal bundle), if the following data are fixed:

(a) a continous mapping $\eta: N \rightarrow B G$;

(b) a normal bundles isomorphism $\Xi: \nu(g) \rightarrow \oplus k \eta^{*}(\gamma)$.

Strictly say, to define «G-structure», a representation of $G$ has to be prescribed. Or, as in [6], a universal bundle $\gamma$ should be fixed. In our case for a group $G$ the only representation is considered, this give no collisions.

Let us consider arbitrary triples $\left(g: N^{n-k d} \uparrow \rightarrow \mathbb{R}^{n}, \eta, \Xi\right)$, where $N$ is closed.

Definition 2. Let us say that triples $\left(g_{0}: N_{0}^{n-k d} \rightarrow \mathbb{R}^{n}, \eta_{0}, \Xi_{0}\right)$ and $\left(g_{1}: N_{1}^{n-k d} \rightarrow \mathbb{R}^{n}, \eta_{1}, \Xi_{1}\right)$ are cobordant, if the following 3 conditions are satisfied:

(i) there exists a compact manifold $W^{n-k d+1}$ and a diffeomorphism $\varphi_{0} \sqcup \varphi_{1}: N_{0} \sqcup N_{1} \rightarrow \partial W$;

(ii) there exists an immersion $G: W^{n-k d+1} \rightarrow \mathbb{R}^{n} \times[0,1]$, which is orthohonal along the boundary $\partial W$ to $\mathbb{R}^{n} \times\{0,1\}$ and which is agree with restrictions of the immersions $g_{0}$ niS $g_{1}$ to $\partial W$, namely, for $s=0,1$ the restriction $\left.G\right|_{\varphi_{s}\left(N_{s}\right)}$ coinsids with the composition $N_{s} \stackrel{\varphi_{s}}{\rightarrow} \mathbb{R}^{n} \rightarrow \mathbb{R}^{n} \times\{s\}$;

\footnotetext{
* National Research University Higher School of Economics, Moscow, Russia; 108840, IZMIRAN, Troitsk, Moscow region, Russia

${ }^{\dagger}$ Moscow State University, Faculty of Mechanics and Mathematics, Leninskie Gory 1, Moscow, 119991 Russia
} 
(iii) there exists a continous mapping $\eta: W^{n-k d+1} \rightarrow B G$ and an isomorphism $\Xi: \nu(g) \rightarrow \oplus k \eta^{*}(\gamma)$, that are agree with $\eta_{s}$ and $\Xi_{s}(s=0,1)$ on the boundary of $W$ in the following natural sence: $\left.\eta\right|_{\varphi_{s}\left(N_{s}\right)}=\eta_{s}$ and $\left.\Xi\right|_{N_{s}}=\Xi_{s}$.

By standard arguments one could proves that the cobordism relation, introduced above, is an equivalence relation. A disjoin union induces an Abelean group structure on the set of cobordism classes, this group is denoted by $\operatorname{Imm}^{G}(n-k d, k d)$. More detailed definition is in $[6$.

In the case when $G$-bundle $\gamma$ is oriented, the manifold $N$ is equipped with a natural orientation, constructed by $\Xi$. In a general case the bundle $\gamma$ is non-oriented, and $N$ is equipped by a natural orientation only in the case $k \equiv 0(\bmod 2)$. Nevertheless, for a non-oriented $\gamma$ and for $k \equiv 1(\bmod 2)$ on $N$ a local orientation is well-defined. Let us consider the details for a special case $G \cong \mathbb{Z}_{4} \rtimes \mathbb{Z}$.

Let $G$ be a group, equipped with an an action of $\mathbb{Z}$ by a (non-trivial) authomorphism $\theta=\theta(1): G \mapsto G$, $1 \in \mathbb{Z}$. The classifying space $B G \rtimes \mathbb{Z}$ is defined as a semi-direct product of $B G$ and the standard circle $S^{1}$, this space is the factor of $B G \times[0,1]$ by identification $B G \times\{0\}$ and $B G \times\{1\}$ using the homeomorphism $B \theta$. The homomorphism $G \rtimes \mathbb{Z} \rightarrow \mathbb{Z}$ classifies the projection $p r_{S}: B G \rtimes \mathbb{Z} \rightarrow S^{1}$, this projection is a locally trivial fibration with the fibre $B G$.

Let us fix an embedding $T=\dot{D}^{n-1} \times S^{1} \subset \mathbb{R}^{n}$ of an open solid torus onto the standard solid torus, which is invariant by rotation trough the coordinate axis. The projection $p r_{T}$ of the solid torus $T$ onto the axial circle $S^{1}$ is well-defined. Denote bypt $\in S^{1}$ a marked point on the circle. Thus, $p r_{S}^{-1}(p t) \subset B G \rtimes \mathbb{Z}$ coincides with $B G \subset B G \rtimes \mathbb{Z}$.

Let us prescribe a representation $G \rtimes \mathbb{Z}$ mïS $\mathbb{R}^{d}$ and the corresponding vector $d$-bundle $\gamma$ over $B G \rtimes \mathbb{Z}$ (the universal bundle).

Definition 3. Let us say that for an immersion $g: N^{n-k d} \rightarrow \mathbb{R}^{n}$ a $G \rtimes \mathbb{Z}$-structure (of the normal bundle of g) is fixed, is the following conditions are satisfied:

(a) the immersion $g$ is an immersion into the solid torus $T \subset \mathbb{R}^{n}$, i.e. is represented by a composition $N^{n-k d} \stackrel{\tilde{g}}{\rightarrow} T \subset \mathbb{R}^{n}$

Denote by $K^{n-k d-1} \subset N^{n-k d}$ a codimension 1 submanifold, which is given by the formula: $\left(p r_{T} \circ g\right)^{-1}(p t)$, assuming, that pt is a regular value for $p_{T} \circ \mathrm{g}$. Denote by $U_{K} \subset N^{n-k d}$ a thin regular neighbourhood of the submanifold $K^{n-k d-1} \subset N^{n-k d}$.

(b) a continuous mapping $\eta: N \rightarrow B G \rtimes \mathbb{Z}$, which induces the corresponding mapping of pairs $\eta:\left(N ; U_{N}, N \backslash\right.$ $\left.U_{N}\right) \rightarrow(B G \rtimes \mathbb{Z} ; B G, B G \rtimes \mathbb{Z} \backslash B G) ;$ moreover, the composition $p_{S} \circ \eta$ is homotopic to a composition pr $_{T} \circ \tilde{g}$, and the homotopy class of this homotopy is given;

(nï) an isomorphism of vector bundles $\Xi: \nu(g) \rightarrow \oplus k \eta^{*}(\gamma)$ is well-defined.

For such mappings with prescribed $G \rtimes \mathbb{Z}$-framing of normal bundle the cobordism relation is well-defined, the definition is analogous to 2 and omitted. On the cobordism classes of $G \rtimes \mathbb{Z}$-framed immersions Abelian group structure is well-defined: the sum of two immersions is defined as the disjoin union of the two parallel copies of immersions in $T$.

Denote this cobordism group by $\operatorname{Imm}^{G \rtimes \mathbb{Z}}(n-k d, k d ; T)$.

\section{Cobordism group $\operatorname{Imm}^{\mathbb{Z}_{4} \rtimes \mathbb{Z}}(n-2 k, 2 k ; T)$}

Consider the group $\mathbb{Z}_{4} \rtimes \mathbb{Z}$. This group is generated by elements $b \in \mathbb{Z}_{4}, \tilde{a} \in \mathbb{Z}$ with the relations $b^{4}=e$, $\tilde{a}^{-1} b \tilde{a}=b^{3}$. A representation $A$ of the group $\mathbb{Z}_{4} \rtimes \mathbb{Z}$ in $\mathbb{R}^{2}$ is given by the formulas:

$$
b \mapsto\left(\begin{array}{cc}
0 & -1 \\
1 & 0
\end{array}\right), \quad \tilde{a} \mapsto\left(\begin{array}{cc}
0 & 1 \\
1 & 0
\end{array}\right) .
$$

Thus, one may consider the group $\operatorname{Imm}_{T}^{\mathbb{Z}_{4} \rtimes \mathbb{Z}}(n-2 k, 2 k ; T)$, which is gepresented by immersions $g: N^{n-2 k} \hookrightarrow$ $T \subset \mathbb{R}^{n}$ with $\mathbb{Z}_{4} \rtimes \mathbb{Z}$-framings.

If $k$ is even, the bundle $\nu(g)$ is oriented (because $w_{1}(\nu(g))=k w_{1}\left(\eta^{*}(\gamma)\right)=0$ even for an non-oriented $\eta^{*}(\gamma)$ ). Then $N$ and $\bar{L}$ are oriented and for prescribed orientations the Herbert formula is satisfied:

$$
j_{*}[\bar{L}]+e_{*}(\nu(g))=0 \text { in the group } H_{n-4 k}(N, \mathbb{Z}) .
$$

In the case $k$ is odd, the considered manifolds, generally speaking, are non-oriented. But, the Herbert formula is generalized using oriented local coefficient system.

Denote on the space $B \mathbb{Z}_{4} \rtimes \mathbb{Z}$ the local coefficients system $\mathbb{Z}^{t w}$ of $\pi_{1}\left(B \mathbb{Z}_{4} \rtimes \mathbb{Z}\right)=\mathbb{Z}_{4} \rtimes \mathbb{Z}$ by authomorphisms of $\mathbb{Z}$ by the following formula:

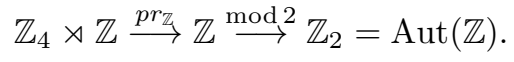


Analogously, the corresponding local system is well-defined for an arbitrary $N^{n-k d}$, which is included into a triple $(g, \eta, \Xi)$, representing an element in $\operatorname{Imm}^{\mathbb{Z}_{4} \rtimes \mathbb{Z}}(n-k d, k d ; T)$. To define the local system the pull-back $\eta^{*}\left(\mathbb{Z}^{t w}\right)$ is used. This local system on $N$ is also denoted by $\mathbb{Z}^{t w}$, this gives no confusion.

In this situation, let us discuss terms in the Herbert formula. Let $\left(g: N^{n-2 k} \rightarrow T \subset \mathbb{R}^{n}, \eta: N \rightarrow B \mathbb{Z}_{4} \rtimes \mathbb{Z}, \Xi\right)$ be a representation of an element in $\operatorname{Imm}^{\mathbb{Z}_{4} \rtimes \mathbb{Z}}(n-2 k, 2 k ; T)$. Double-point manifold of $\bar{L}$ is oriented. This follows from the formula: $\nu\left(\bar{L} \leftrightarrow \mathbb{R}^{n}\right)=\left.\nu\left(\bar{N} \leftrightarrow \mathbb{R}^{n}\right)\right|_{\bar{L}} \oplus \nu(\bar{L} \leftrightarrow N)=\left.\left.\nu(g)\right|_{\bar{L}} \oplus \tau^{*} \nu(g)\right|_{\bar{L}}$, where $\tau: \bar{L} \rightarrow \bar{L}$ is the standard involution, which is induced by permutation of coordinates in $N \times N$. The oriented classes $w_{1}$ of the bundles $\left.\nu(g)\right|_{\bar{L}}$ and $\left.\tau^{*} \nu(g)\right|_{\bar{L}}$ coincide, because a closed loop $\gamma$ in $\bar{L}$ changes the orientation of the fibre $\left.\nu(g)\right|_{\bar{L}}$, iff the loop $\tau \circ \gamma$ also changes the orientation. Thus, one may define the fundamental class $[\bar{L}] \in H_{n-4 k}(\bar{L}, \mathbb{Z})$. This proves that the first term $i_{\bar{L} \rightarrow N}([\bar{L}]) \in H_{n-4 k}(N, \mathbb{Z})$ in the formula is well-defined.

Let us consider the second term. The main difficult concerns to non-orientable bundles. But in the considered case this problem is solved. Denote by $o(\nu(g))$ the orienting system (the locally coefficients system with coefficients $\mathbb{Z}$ ) of the bundle $o(\nu(g))$. Recall, that on the manifold $N$ a local coefficient $\mathbb{Z}$-system is given by the corresponding element $\operatorname{Hom}\left(\pi_{1}(N)\right.$, Aut $\left.(\mathbb{Z})\right)=H^{1}\left(N, \mathbb{Z}_{2}\right)$. The oriented system $o(\nu(g))$ is given by the class $w_{1}(\nu(g)) \in H^{1}\left(N, \mathbb{Z}_{2}\right)$. For a bundle $\nu(g)$ the Euler class $e(\nu(g)) \in H^{2 k}(N, o(\nu(g)))$ is well-defined. Alternatively, the manifold $N$ is oriented with the local coefficients system $o(T N)$. Using the decomposition $T N \oplus \nu(g)=n \varepsilon_{N}$, where $\varepsilon_{N}$ is the trivial line bundle over $N$, the isomorphism $o(T N)=o(\nu(g))$ is well-defined. Thus, the fundamental class $[N] \in H_{n-2 k}(N, o(\nu(g)))$ is well-defined. Obviously, $o(\nu(g))=\left(\eta^{*}\left(\mathbb{Z}^{t w}\right)\right)^{\otimes k}$. Then $H_{n-2 k}(N, o(\nu(g)))=H_{n-2 k}\left(N,\left(\eta^{*}\left(\mathbb{Z}^{t w}\right)\right)^{\otimes k}\right)$. From the last formula the Euler homology class $e_{*}(\nu(g)) \in H_{n-4 k}(N, \mathbb{Z})$ is defined by $[N] \cap e(\nu(g))=[N] \cap\left(e\left(\eta^{*}(\gamma)\right)\right)^{k}$.

Theorem 1. Assume that the manifold $N$ is equipped by $\mathbb{Z}_{4} \rtimes \mathbb{Z}$-framing. Then in the group $H_{n-4 k}(N, \mathbb{Z})$ the following formula is well-defined:

$$
j_{*}[\bar{L}]+e_{*}(\nu(g))=0 .
$$

Proof. The proof follows from a straightforward generalisation of classical arguments [1].

Remark 1. Obviously, the theorem is generalised for $G \rtimes Z$-framing, in the case of representation $G \rightarrow S O(d)$, which is extended to a representation $G \rtimes \mathbb{Z} \rightarrow O(d)$

\section{Hurewicz homomorphim}

Define the Hurewicz homomorphism

$$
H: \operatorname{Imm}^{\mathbb{Z} / 4 \rtimes \mathbb{Z}}(n-2 k, 2 k ; T) \rightarrow H_{n-2 k}\left(B \mathbb{Z} / 4 \rtimes \mathbb{Z} ;\left(\mathbb{Z}^{t w}\right)^{\otimes k}\right) .
$$

Take an $x \in \operatorname{Imm}^{\mathbb{Z} / 4 \rtimes \mathbb{Z}}(n-2 k, 2 k ; T)$, which is represented by a triple $\left(g: N^{n-2 k} \rightarrow T \subset \mathbb{R}^{n}, \eta: N \rightarrow\right.$ $\left.B \mathbb{Z}_{4} \rtimes \mathbb{Z}, \Xi\right)$. The fundamental class $[N] \in H_{n-2 k}\left(N ; \eta^{*}\left(\left(\mathbb{Z}^{t w}\right)^{\otimes k}\right)\right)$ is well-defined. Define $H(x)=\eta_{*}([N])$. Obviously, this class depends not of a representation of $x$.

Lemma 1. (a) The mapping $i: B \mathbb{Z}_{4} \subset B \mathbb{Z}_{4} \rtimes \mathbb{Z}$ of the classified spaces, corresponded to the monomorphism $\mathbb{Z}_{4} \subset \mathbb{Z}_{4} \rtimes \mathbb{Z}$, induces the morphism $i^{*}\left(\mathbb{Z}^{t w}\right)=\mathbb{Z}$ of local coefficients systems.

(b) Assuming $q=4 m+1$, the isomorphism is well-defined:

$$
i_{*}: \mathbb{Z}_{4}=H_{q}(B \mathbb{Z} / 4 ; \mathbb{Z}) \cong H_{q}\left(B \mathbb{Z} / 4 ; i^{*}\left(\mathbb{Z}^{t w}\right)\right) \rightarrow H_{q}\left(B \mathbb{Z} / 4 \rtimes \mathbb{Z} ; \mathbb{Z}^{t w}\right) .
$$

(c) Assuming $q=4 m+3$, the isomorphism is well-defined:

$$
i_{*}: \mathbb{Z}_{4}=H_{q}(B \mathbb{Z} / 4 ; \mathbb{Z}) \rightarrow H_{q}(B \mathbb{Z} / 4 \rtimes \mathbb{Z} ; \mathbb{Z}) .
$$

(d) $i^{*}: H^{2}\left(B \mathbb{Z} / 4 \rtimes \mathbb{Z} ; \mathbb{Z}^{t w}\right) \rightarrow H^{2}(B \mathbb{Z} / 4 ; \mathbb{Z})=\mathbb{Z}_{4}$ is isomorphism, and the Euler class $e=e(\gamma)$ of the universal bundle $\gamma$ is a generator. Moreover, the formula $\cap e \mapsto-\cap$ e determines isomorphisms $H_{4 m+1}\left(B \mathbb{Z}_{4} \rtimes\right.$ $\left.Z ; \mathbb{Z}^{t w}\right) \rightarrow H_{4 m-1}\left(B \mathbb{Z}_{4} \rtimes Z ; \mathbb{Z}\right)$ and $H_{4 m-1}\left(B \mathbb{Z}_{4} \rtimes \mathbb{Z} ; \mathbb{Z}\right) \rightarrow H_{4 m-3}\left(B \mathbb{Z}_{4} \rtimes \mathbb{Z} ; \mathbb{Z}^{t w}\right)$ for $m \geq 1$.

Proof. Recall, that $H_{q}(B \mathbb{Z} / 4 ; \mathbb{Z})=\mathbb{Z}_{4}$ for an arbitrary odd $q \geq 1$, and $H_{0}(B \mathbb{Z} / 4 ; \mathbb{Z})=\mathbb{Z}$. Homology of the group $\mathbb{Z}$ in a module $M$ are known: $H_{0}(B \mathbb{Z}, M)=M_{\mathbb{Z}}$ (coinvariants of the $\mathbb{Z}$-action on $\left.M\right)$ and $H_{1}(\mathbb{Z}, M)=M^{\mathbb{Z}}$ (invariants of the $\mathbb{Z}$-action on $M$ ).

Consider the space $E \mathbb{Z}_{4} \rtimes \mathbb{Z}$, this space is realized as $S^{\infty} \times \mathbb{R}$. Here we have $S^{\infty}=\bigcup_{n}\left\{\left(z_{1}, \ldots, z_{n}\right) \in\right.$ $\left.\left.\mathbb{C}^{n}\left|\sum\right| z_{k}\right|^{2}=1\right\}$. The action of the generators $b, \tilde{a}$ of the group $\mathbb{Z}_{4} \rtimes \mathbb{Z}$ are given by the formulas: $b(z, t)=$ $\left(e^{\pi i / 2} z, t\right)$, and $\tilde{a}(z, t)=(\bar{z}, t+1)$. It is easy to see that the relations $b^{4}=1$, and $\tilde{a}^{-1} b \tilde{a}=b^{3}$ are satisfied, the corresponding (free) action of $\mathbb{Z}_{4} \rtimes \mathbb{Z}$ on $E \mathbb{Z}_{4} \rtimes \mathbb{Z}=S^{\infty} \times \mathbb{R}$ is well-defined. 
Consider the spectral sequence of the bundle $B \mathbb{Z}_{4} \rightarrow B \mathbb{Z}_{4} \rtimes \mathbb{Z} \rightarrow B \mathbb{Z}$ for the trivial module $\mathbb{Z}$ of coefficients. The second term of this sequence $E_{p q}^{2}=H_{p}\left(B \mathbb{Z}, H_{q}\left(B \mathbb{Z}_{4}, \mathbb{Z}\right)\right)$. By dimension reason, $E_{* *}^{2}=E_{* *}^{\infty}$. Non-trivial terms could be only $E_{0 q}^{2}$ and $E_{1, q-1}^{2}$ for odd $q \geq 1$ and for $q=0$. Therefore, the problem is to calculate the action of the generator $\tilde{a} \in \mathbb{Z}$ in homology $H_{q}\left(B \mathbb{Z}_{4}, \mathbb{Z}\right)$.

Denote the corresponding homomorphism by $\tilde{a}_{*}$. Because non-trivial groups $H_{q}\left(B \mathbb{Z} \mathbb{Z}_{4}, \mathbb{Z}\right)$ are isomorphic to $\mathbb{Z}$, or to $\mathbb{Z}_{4}$, the action $\tilde{a}_{*}$ in homologies $H_{q}\left(B \mathbb{Z}_{4}, \mathbb{Z}\right)$ is the multiplication on \pm 1 . The action of the generator $\tilde{a}$ coincides to the conjugation of all coordinates in $S^{\infty}$, therefore the sign depends of $q$ as follows: $\tilde{a}_{*}=1$ for $q=4 m+3$, and $\tilde{a}_{*}=-1$ for $q=4 m+1$. Therefore $E_{0 q}^{2}$ and $E_{1 q}^{2}$ are isomorphic to $\mathbb{Z}_{4}$ for $q=4 m+3$ and to $\mathbb{Z}_{2}$ for $q=4 m+1$. Statement (a) is proved.

Calculation of cohomologies $H_{*}\left(B \mathbb{Z}_{4} \rtimes \mathbb{Z}, \mathbb{Z}^{t w}\right)$ are analogous. Let us note, that to calculate the action of $\mathbb{Z}$ in homologies $H_{q}\left(B \mathbb{Z}_{4}, i^{*}\left(\mathbb{Z}^{t w}\right)\right)$ an additional sign -1 is required, because the generator $\tilde{a}$ acts in the module of the coefficients by multiplication on -1 . Therefore, we get: $\tilde{a}_{*}=1$ for $q=4 m+1$ and $\tilde{a}_{*}=-1$ for $q=4 m+3$. The terms $E_{0 q}^{2}$ пїS $E_{1 q}^{2}$ are isomorphic to $\mathbb{Z}_{4}$ for $q=4 m+1$ and to $\mathbb{Z}_{2}$ for $q=4 m+3$. Proofs of the last statements are analogous.

\section{Self-intersection manifolds of $\mathbb{Z} / 4 \rtimes \mathbb{Z}$-framed immersions}

Let $(g, \eta, \Xi)$ be a $\mathbb{Z} / 4 \rtimes \mathbb{Z}$-framed immersion. The self-intersection manifold $L^{n-4 k}$ of the immersion $g$ is defined as $L=\bar{L} / \tau$, where $\tau$ is the coordinate involution on $N \times N$. The 2 -sheeted covering $\pi: \bar{L} \rightarrow L$, which is called the canonical covering, is well-defined. The following conditions are satisfied.

(1) There exists an immersion $h: L^{n-4 k} \rightarrow T \subset \mathbb{R}^{n}$, for which 2-sheeted covering $\bar{L}^{n-4 k}$ is included in the following diagram,

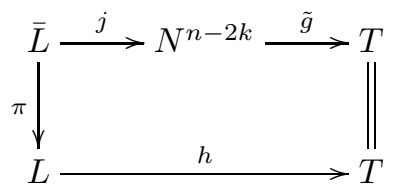

In this diagram the horizontal mappings are immersions. Recall, that the immersion $j: \bar{L} \rightarrow N$ is defined by composition of the embedding $\bar{L} \subset N \times N$ and the projection $N \times N \rightarrow N$ onto the first factor.

(2) The normal bundle over $\bar{L}$ is decomposed into 2 factors $\left.\left.\nu(g)\right|_{\bar{L}} \oplus \tau * \nu(g)\right|_{\bar{L}}$, where $\tau: \bar{L} \rightarrow \bar{L}$ is the involution, which permutes the sheets of the double covering. Therefore the normal bundle over $\bar{h}: \bar{L} \rightarrow T$ is decomposed into the Whitney sum of $k$ isomorphic copies of the bundle $\eta \oplus \tau^{*} \eta$, each copy is classified by a mapping in $B\left(\mathbb{Z}_{4} \rtimes \mathbb{Z}\right) \times\left(\mathbb{Z}_{4} \rtimes \mathbb{Z}\right)$. Because $\tilde{h}$ and $\tilde{h} \circ \tau$ coincide by definition of the manifold $\bar{L}$, compositions $\left.\nu\right|_{\bar{L}}$ and $\left.\tau^{*} \nu\right|_{\bar{L}}$ with the projection $p r_{S}: B \mathbb{Z}_{4} \rtimes Z \rightarrow B \mathbb{Z}$ coincide. Therefore, the classifying mapping of the bundle $\eta \oplus \tau^{*} \eta$, is decomposed using the mapping $B\left(\mathbb{Z}_{4} \times \mathbb{Z}_{4}\right) \rtimes \mathbb{Z} \rightarrow B\left(\mathbb{Z}_{4} \rtimes \mathbb{Z}\right) \times\left(\mathbb{Z}_{4} \rtimes \mathbb{Z}\right)$, which is induced by the diagonal inclusion $\mathbb{Z} \subset \mathbb{Z} \times \mathbb{Z}$. This shows that the normal bundle over $h: L \uparrow T \subset \mathbb{R}^{n}$ is decomposed into the Whitney sum of $k$ copies of a 4-dimensional bundle, which is classified by the mapping $\zeta: L \rightarrow B\left(\left(\mathbb{Z}_{4} \times \mathbb{Z}_{4}\right) \rtimes \mathbb{Z}\right) \rtimes \mathbb{Z}_{2}$. The corresponding universal bundle over $B\left(\left(\mathbb{Z}_{4} \times \mathbb{Z}_{4}\right) \rtimes \mathbb{Z}\right) \rtimes \mathbb{Z}_{2}$ will be denoted by $\gamma^{[2]}$ for short. Let us note, that $\left(\left(\mathbb{Z}_{4} \times \mathbb{Z}_{4}\right) \rtimes \mathbb{Z}\right) \rtimes \mathbb{Z}_{2}=\left(\left(\mathbb{Z}_{4} \times \mathbb{Z}_{4}\right) \rtimes \mathbb{Z}_{2}\right) \rtimes \mathbb{Z}$, because the actions of $\mathbb{Z}$ and $\mathbb{Z}_{2}$

on $\mathbb{Z}_{4} \times \mathbb{Z}_{4}$ are commuted with each other (a generator of $\mathbb{Z}$ multiplies the both generators elements of $\mathbb{Z}_{4} \times \mathbb{Z}_{4}$ by -1 , the generator in $\mathbb{Z}_{2}$ changes the above generators; the action between $\mathbb{Z}$ and $\mathbb{Z}_{2}$ is trivial).

Thus, the triple $(g, \eta, \Xi)$ with a $\mathbb{Z}_{4} \rtimes \mathbb{Z}$-framing determines a triple $(h, \zeta, \Lambda)$, where $h: L^{n-4 k} \rightarrow \mathbb{R}^{n}$ is an immersion, $\zeta: L \rightarrow B\left(\left(\mathbb{Z}_{4} \times \mathbb{Z}_{4}\right) \rtimes \mathbb{Z}\right) \rtimes \mathbb{Z}_{2}$ is a continous mapping and $\Lambda: \nu(h) \rightarrow k \zeta^{*}\left(\gamma^{[2]}\right)$ is an isomorphism.

\section{Subgroups $\left(\mathbb{Z}_{4} \times \mathbb{Z}_{4}\right) \rtimes \mathbb{Z}_{2}$ and $\left(\left(\mathbb{Z}_{4} \times \mathbb{Z}_{4}\right) \rtimes \mathbb{Z}_{2}\right) \rtimes \mathbb{Z}$ and its representation in $S O(4)$}

The representation $A^{[2]}$ of the group $\left(\left(\mathbb{Z}_{4} \times \mathbb{Z}_{4}\right) \rtimes \mathbb{Z}_{2}\right) \rtimes Z$ in $O(4)$ is given by the formulas:

$$
\begin{array}{ll}
b_{1}=(((1,0), 0), 0) \mapsto\left(\begin{array}{cccc}
0 & -1 & 0 & 0 \\
1 & 0 & 0 & 0 \\
0 & 0 & 1 & 0 \\
0 & 0 & 0 & 1
\end{array}\right), & b_{2}=(((0,1), 0), 0) \mapsto\left(\begin{array}{cccc}
1 & 0 & 0 & 0 \\
0 & 1 & 0 & 0 \\
0 & 0 & 0 & -1 \\
0 & 0 & 1 & 0
\end{array}\right), \\
t=(((0,0), 1), 0) \mapsto\left(\begin{array}{cccc}
0 & 0 & 1 & 0 \\
0 & 0 & 0 & 1 \\
1 & 0 & 0 & 0 \\
0 & 1 & 0 & 0
\end{array}\right), & \tilde{a}=(((0,0), 0), 1) \mapsto\left(\begin{array}{cccc}
0 & 1 & 0 & 0 \\
1 & 0 & 0 & 0 \\
0 & 0 & 0 & 1 \\
0 & 0 & 1 & 0
\end{array}\right) .
\end{array}
$$




\section{Subgroup $\mathbf{Q} \subset\left(\mathbb{Z}_{4} \times \mathbb{Z}_{4}\right) \rtimes \mathbb{Z}_{2}$}

By $\mathbf{Q}$ is denoted the standard integer quaternion group, generated by $\pm 1, \pm \mathbf{i}, \pm \mathbf{j}, \pm \mathbf{k}$, this group is included into $\left(\mathbb{Z}_{4} \times \mathbb{Z}_{4}\right) \rtimes \mathbb{Z}_{2}$ using the correspondence:

$$
1 \mapsto((0,0), 0), \quad \mathbf{i} \mapsto((1,-1), 0), \quad \mathbf{j} \mapsto((1,1), 1), \quad \mathbf{k} \mapsto((2,0), 1)
$$

Then the standard representation of the group $\mathbb{Z}_{4}$ in $\mathbb{R}^{2}$ by rotations trough angles divided by $\pi / 2$, induces the representation of the group $\left(\mathbb{Z}_{4} \times \mathbb{Z}_{4}\right) \rtimes \mathbb{Z}_{2}$ in $\mathbb{R}^{4}$. This representation maps the generators of $\mathbf{Q}$ by the following formulas:

$$
\mathbf{i} \mapsto\left(\begin{array}{cccc}
0 & -1 & 0 & 0 \\
1 & 0 & 0 & 0 \\
0 & 0 & 0 & 1 \\
0 & 0 & -1 & 0
\end{array}\right), \quad \mathbf{j} \mapsto\left(\begin{array}{cccc}
0 & 0 & 0 & -1 \\
0 & 0 & 1 & 0 \\
0 & -1 & 0 & 0 \\
1 & 0 & 0 & 0
\end{array}\right), \quad \mathbf{k} \mapsto\left(\begin{array}{cccc}
0 & 0 & -1 & 0 \\
0 & 0 & 0 & -1 \\
1 & 0 & 0 & 0 \\
0 & 1 & 0 & 0
\end{array}\right) .
$$

Subgroups $\mathbb{Z}_{4} \times \mathbb{Z}_{2} \subset\left(\mathbb{Z}_{4} \times \mathbb{Z}_{4}\right) \rtimes \mathbb{Z}_{2}$ and $\left(\mathbb{Z}_{4} \times \mathbb{Z}_{2}\right) \rtimes \mathbb{Z} \subset\left(\left(\mathbb{Z}_{4} \times \mathbb{Z}_{4}\right) \rtimes \mathbb{Z}_{2}\right) \rtimes \mathbb{Z}$

The standard diagonal mapping $\mathbb{Z}_{4} \rightarrow \mathbb{Z}_{4} \times \mathbb{Z}_{4}$ induces an inclusion $\mathbb{Z} / 4 \times \mathbb{Z} / 2$ onto a subgroup in $(\mathbb{Z} / 4 \times \mathbb{Z} / 4) \rtimes$ $\mathbb{Z} / 2$. The generators $b, t$ of the group $\mathbb{Z} / 4 \times \mathbb{Z} / 2$ are mapped into the elements $((1,1), 0)$ and $((0,0), 1)$ of the group $(\mathbb{Z} / 4 \times \mathbb{Z} / 4) \rtimes \mathbb{Z} / 2$ therefore this elements are represented by the following formulas:

$$
b \mapsto\left(\begin{array}{cccc}
0 & -1 & 0 & 0 \\
1 & 0 & 0 & 0 \\
0 & 0 & 0 & -1 \\
0 & 0 & 1 & 0
\end{array}\right), \quad t \mapsto\left(\begin{array}{cccc}
0 & 0 & 1 & 0 \\
0 & 0 & 0 & 1 \\
1 & 0 & 0 & 0 \\
0 & 1 & 0 & 0
\end{array}\right)
$$

The subgroup $\mathbb{Z} / 4 \times \mathbb{Z} / 2 \rtimes \mathbb{Z} \subset((\mathbb{Z} / 4 \times \mathbb{Z} / 4) \rtimes \mathbb{Z} / 2) \rtimes \mathbb{Z}$ is defined as extension of the considered subgroup by he inclusion $(\mathbb{Z} / 4 \times \mathbb{Z} / 4) \rtimes \mathbb{Z} / 2 \subset((\mathbb{Z} / 4 \times \mathbb{Z} / 4) \rtimes \mathbb{Z} / 2) \rtimes \mathbb{Z}$.

\section{Cap-product with 2-dimensional class}

For a representation $\left(g: N^{n-2 k} \rightarrow \mathbb{R}^{n}, \eta, \Xi\right)$ of a prescribed class in $\operatorname{Imm}^{\mathbb{Z} / 4 \rtimes \mathbb{Z}}(n-2 k, 2 k ; T)$ the bundle $\eta^{*}(\gamma)$ is well-defined. Trivial vectors of a generic section form a submanifold $N_{1} \subset N$, moreover, for the image of the fundamental class $\left[N_{1}\right] \in H_{n-2 k-2}\left(N_{1},\left(\mathbb{Z}^{t w}\right)^{\otimes(k+1)}\right)$ by the embedding $i_{N}: N_{1} \rightarrow N$ the following formula is satisfied: $\left(i_{N}\right)_{*}\left[N_{1}\right]=[N] \cap \eta^{*}(e)$ in the group $H_{n-2 k-2}\left(N,\left(\mathbb{Z}^{t w}\right)^{\otimes(k+1)}\right)$. Therefore, in the group $H_{n-2 k-2}\left(B \mathbb{Z}_{4} \rtimes \mathbb{Z},\left(\mathbb{Z}^{t w}\right)^{\otimes(k+1)}\right)$ the following equation is satisfied: $H\left(N_{1}\right)=H(N) \cap e\left(\right.$ or, $\eta_{*} \circ\left(i_{N}\right)_{*}\left[N_{1}\right]=$ $\left.\eta_{*}[N] \cap e\right)$.

\section{Manifolds of self-intersection points of the immersions $\bar{L}_{1} \subset N_{1}$ and $\bar{L} \subset N$}

Recall, in the Introduction the manifold $\bar{L}$ is defined by the formula:

$$
\bar{L}=\{(x, y) \in N \times N \mid x \neq y, g(x)=g(y)\},
$$

and the immersion $j: \bar{L} \subset N \times N \stackrel{p r_{1}}{\rightarrow} N$ is well-defined. There exists a 2-sheeted covering $\pi: \bar{L} \rightarrow L$, where $L=\bar{L} / \tau$, and $\tau$ is the involution on $N \times N$, which permutes the factors: $\tau(x, y)=(y, x)$. The manifold $L_{1}$ and its 2-sheeted covering $\bar{L}_{1} \subset N_{1} \times N_{1}$ are defined analogously. Denote by $i_{L}$ the inclusion of $L_{1}$ into $L$.

Using the Herbert Theorem for immersions $g: N^{n-2 k} \rightarrow \mathbb{R}^{n}$ חïS $g_{1}: N^{n-2 k-2} \rightarrow \mathbb{R}^{n}$, we get the following 2 relations:

$$
\begin{aligned}
& j_{*}[\bar{L}]+[N] \cap \eta^{*}(e)^{k}=0 \text { пїS } H_{n-4 k}\left(N,\left(\mathbb{Z}^{t w}\right)^{\otimes 2 k}\right), \\
& \left(j_{1}\right)_{*}\left[\bar{L}_{1}\right]+\left[N_{1}\right] \cap \eta^{*}(e)^{(k+1)}=0 \text { пїS } H_{n-4 k-4}\left(N,\left(\mathbb{Z}^{t w}\right)^{\otimes(2 k+2)}\right) .
\end{aligned}
$$

Let us apply the homomorphism $\left(i_{N}\right)_{*}$ for elements of the formula, in the group $H_{n-4 k-4}\left(N,\left(\mathbb{Z}^{t w}\right)^{2 k+2}\right)$ we get the formula:

$$
j_{*}[\bar{L}] \cap \eta^{*}\left(e^{2}\right)=-[N] \cap \eta^{*}(e)^{k+2}=-\left(i_{N}\right)_{*}\left[N_{1}\right] \cap \eta^{*}(e)^{k+1}=\left(i_{N}\right)_{*} \circ\left(j_{1}\right)_{*}\left[\bar{L}_{1}\right] .
$$

Let us calculate the element $\left(i_{L}\right)_{*}\left[\bar{L}_{1}\right]$ in the group $H_{n-4 k-4}\left(\bar{L},\left(\mathbb{Z}^{t w}\right)^{\otimes(2 k+2)}\right)$ by an alternative way. Obviously, the manifold $\bar{L}_{1}$ is the intersection of the two submanifolds $\bar{L} \cap N_{1}$ and $\tau\left(\bar{L} \cap N_{1}\right)$, where $\tau: \bar{L} \rightarrow \bar{L}$ is the involution, which permutes the sheets of the covering $\bar{L} \rightarrow L$. From this fact we get in the group $H_{n-4 k-4}\left(L,\left(\mathbb{Z}^{t w}\right)^{\otimes(2 k+2)}\right)$ the following relation: $\left(i_{L}\right)_{*}\left[L_{1}\right]=[\bar{L}] \cap\left(\left.e\right|_{\bar{L}} \cup \tau^{*}\left(\left.e\right|_{\bar{L}}\right)\right)$, where $\left.e\right|_{L}=\left(\bar{\zeta} \circ p r_{1}\right)^{*}(e)$. In a general case the classes $\tau^{*}\left(\left.e\right|_{\bar{L}}\right)$ and $\left.e\right|_{\bar{L}}$ are related unpredictable way. But, in a several cases, when 
the structured group of the normal bundle over $L$ is reduced to a special subgroup, this relation is described explicitly.

Consider the commutative dyagrame:

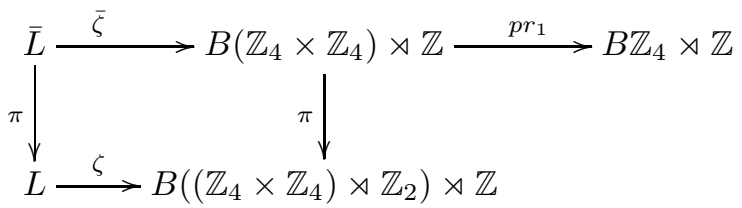

By the commutativity, relationship between $\left.e\right|_{L}$ and $\tau^{*}\left(\left.e\right|_{L}\right)$ is determined, using relationship between $\left(p r_{1}\right)^{*}(e)$ and $\tau^{*}\left(\left(p r_{1}\right)^{*}(e)\right)$ in the cohomologies of the classifying space $B\left(\mathbb{Z}_{4} \times \mathbb{Z}_{4}\right) \rtimes \mathbb{Z}$. This is done in the following two lemmas.

Consider the mappings of the classifying spaces:

$$
\begin{gathered}
I_{1}: B Q \subset B\left(\mathbb{Z}_{4} \times \mathbb{Z}_{4}\right) \rtimes \mathbb{Z}_{2} \subset B\left(\left(\mathbb{Z}_{4} \times \mathbb{Z}_{4}\right) \rtimes \mathbb{Z}_{2}\right) \rtimes \mathbb{Z}, \\
I_{2}: B\left(\mathbb{Z}_{4} \times \mathbb{Z}_{2}\right) \rtimes \mathbb{Z} \subset B\left(\left(\mathbb{Z}_{4} \times \mathbb{Z}_{4}\right) \rtimes \mathbb{Z}_{2}\right) \rtimes \mathbb{Z},
\end{gathered}
$$

which are induced by inclusions onto the subgroups. By $\bar{I}_{1}$ riï $\bar{I}_{2}$ let us denote the corresponding mappings of the 2-sheeted coverings over this spaces

$$
\begin{gathered}
\bar{I}_{1}: B \mathbb{Z}_{4} \rightarrow B\left(\mathbb{Z}_{4} \times \mathbb{Z}_{4}\right) \rtimes \mathbb{Z}, \\
\bar{I}_{2}: B \mathbb{Z}_{4} \times \rtimes \mathbb{Z} \rightarrow B\left(\mathbb{Z}_{4} \times \mathbb{Z}_{4}\right) \rtimes \mathbb{Z} .
\end{gathered}
$$

Put $e_{0}=\left(p r_{1}\right)^{*}(e), e_{Q}=\left(\bar{I}_{1}\right)^{*}\left(e_{0}\right), e_{\text {diag }}=\left(\bar{I}_{2}\right)^{*}\left(e_{0}\right)$.

Lemma 2. The following formula is satisfied: $\tau^{*}\left(e_{Q}\right)=-e_{Q}$.

Proof. Let us realized the space $B Q$ as the factor of the quaternion units sphere $S_{\mathbb{H}}^{\infty} / Q=S_{\mathbb{H}}^{\infty} /\{ \pm, 1, \pm \mathbf{i}, \pm \mathbf{j}, \pm \mathbf{k}\}$, and the space $B \mathbb{Z}_{4}$ as the quotient of the considered sphere over the cyclic subgroup, generated by the quaternion unit i: $S_{\mathbb{H}}^{\infty} / \mathbb{Z}_{4}=S_{\mathbb{H}}^{\infty} /\{ \pm, 1, \pm \mathbf{i}\}$. In this case the involution $\tau: S_{\mathbb{H}}^{\infty} / \mathbb{Z}_{4} \rightarrow S_{\mathbb{H}}^{\infty} / \mathbb{Z}_{4}$, which permutes the sheets of the covering $B \mathbb{Z}_{4} \rightarrow B Q$, corresponds to the multiplication on the quaternion unit $\mathbf{j}$.

It is convenient to investigate the action $\tau$ not on the 2-generator $i^{*}\left(e_{Q}\right)$ of the cohomology group of the space $B \mathbb{Z}_{4}$, but on the generator $\alpha$ of 1-dimensional homology group, which corresponds to $i^{*}\left(e_{Q}\right)$ by the Universal Coefficients Theorem. The generator $\alpha$ is represented by the loop $(\cos t+\mathbf{i} \sin t) x$, where $t \in[0, \pi / 2]$ and $x$ is an arbitrary point in $S_{\mathbb{H}}^{\infty} /\{ \pm, 1, \pm \mathbf{i}\}$. Two such loops determines the same class $\alpha$. Therefore, the class $\alpha$ represents by the loop $(\cos t+\mathbf{i} \sin t) x$, as well as by the loop $\gamma(t)=(\cos t+\mathbf{i} \sin t) \mathbf{j} x$, the last representative is more convenient to investigate the element $\tau_{*}(\alpha)$. Because the action $\tau$ corresponds to the multiplication on $\mathbf{j}$, the element $\tau_{*}(\alpha)$ is represented by the loop $\gamma_{2}(t)=\mathbf{j}(\cos t+\mathbf{i} \sin t) x$. Obviously, $\gamma_{1}(t)$ is given by $\gamma_{2}(t)$ by the reversing of the orientation, therefore, $\tau_{*}(\alpha)=-\alpha$.

Corollary 1. Let an element $\bar{x} \in H_{q}\left(B \mathbb{Z}_{4} \times \mathbb{Z}_{4} \rtimes \mathbb{Z},\left(\mathbb{Z}^{t w}\right)^{\otimes k}\right)$ belongs to the image $\left(\bar{I}_{1}\right)_{*}$. Then for $\bar{x} \cap \tau^{*}\left(e_{0}\right) \in$ $H_{q-2}\left(B \mathbb{Z}_{4} \times \mathbb{Z}_{4} \rtimes \mathbb{Z},\left(\mathbb{Z}^{t w}\right)^{\otimes k-1}\right)$ the following equation is satisfied: $\bar{x} \cap \tau^{*}\left(e_{0}\right)=-\bar{x} \cap e_{0}$.

Proof. Let $\bar{x}=\left(\bar{I}_{1}\right)_{*}(u)$. Then we have

$$
\bar{x} \cap \tau^{*}\left(e_{0}\right)=\left(\bar{I}_{1}\right)_{*}(u) \cap \tau^{*}\left(e_{0}\right)=\left(\bar{I}_{1}\right)_{*}\left(u \cap \tau^{*}\left(I_{1}^{*}\left(e_{0}\right)\right)\right)=
$$

$$
=\left(I_{1}\right)_{*}\left(u \cap \tau^{*}\left(e_{Q}\right)\right)=\left(I_{1}\right)_{*}\left(u \cap\left(-e_{Q}\right)\right) .
$$

By analogous calculations, we get the equation: $\bar{x} \cap e_{0}=\left(I_{1}\right)_{*}\left(u \cap e_{Q}\right)$.

Lemma 3. The following equaton is satisfied: $\tau^{*}\left(e_{\text {diag }}\right)=e_{\text {diag }}$.

Proof. Let us calculate the action $\tau^{*}$ on the generator of the group $H^{2}\left(B \mathbb{Z}_{4} \rtimes \mathbb{Z}, \mathbb{Z}^{t w}\right)=\mathbb{Z}_{4}$. Obviously, the covering $B \mathbb{Z}_{4} \rtimes \mathbb{Z} \rightarrow B \mathbb{Z}_{4} \times \mathbb{Z}_{2} \rtimes \mathbb{Z}$ is represented as $B \mathbb{Z}_{4} \rtimes \mathbb{Z} \times S^{\infty} \stackrel{i d \times \pi}{\longrightarrow} B \mathbb{Z}_{4} \rtimes \mathbb{Z} \times\left(S^{\infty} / \mathbb{Z}_{2}\right)$. Therefore, the involution $\tau$, which permutes sheets of the double covering, acts in (co)homology of the space $B \mathbb{Z}_{4} \rtimes \mathbb{Z}$ by the identity.

Corollary 2. Assume that $\bar{y} \in H_{q}\left(B \mathbb{Z}_{4} \times \mathbb{Z}_{4} \rtimes \mathbb{Z},\left(\mathbb{Z}^{t w}\right)^{\otimes k}\right)$ belongs to the image of the homomorphism $\left(\bar{I}_{2}\right)_{*}$.

(a) Then for $\bar{y} \cap \tau^{*}\left(e_{0}\right) \in H_{q-2}\left(B \mathbb{Z}_{4} \times \mathbb{Z}_{4} \rtimes \mathbb{Z}\right.$, $\left.\left(\mathbb{Z}^{t w}\right)^{\otimes k-1}\right)$ the following equality is satisfied: $\bar{y} \cap \tau^{*}\left(e_{0}\right)=\bar{y} \cap e_{0}$

(b) Additionally, if $\bar{y}$ belong to the image of the transfer homomorphism of the 2-sheeted covering, then $\bar{y}$ is an even element. 
Proof. Assume that $\bar{y}=\left(\bar{I}_{2}\right)_{*}(v)$. Then we get:

$$
\bar{y} \cap \tau^{*}\left(e_{0}\right)=\left(\bar{I}_{2}\right)_{*}(v) \cap \tau^{*}\left(e_{0}\right)=\left(\bar{I}_{2}\right)_{*}\left(v \cap \tau^{*}\left(I_{2}^{*}\left(e_{0}\right)\right)\right)=
$$

$$
=\left(I_{2}\right)_{*}\left(u \cap \tau^{*}\left(e_{\text {diag }}\right)\right)=\left(I_{2}\right)_{*}\left(u \cap e_{\text {diag }}\right) .
$$

By analogous calculations, we get the equation $\bar{y} \cap e_{0}=\left(I_{2}\right)_{*}\left(v \cap e_{\text {diag }}\right)$.

To prove the statement (b), let us note, that the factor-homomorphism $\mathbb{Z}_{4} \times \mathbb{Z}_{2} \rtimes \mathbb{Z} \rightarrow \mathbb{Z}_{4} \rtimes \mathbb{Z}$ determines the mapping $s: B \mathbb{Z}_{4} \times \mathbb{Z}_{2} \rtimes \mathbb{Z} \rightarrow B \mathbb{Z}_{4} \rtimes \mathbb{Z}$, for which the composition $s \circ \pi$ is homotopic to the identity. Then $\bar{y}=s_{*}\left(\pi_{*}(\bar{y})\right)$. The element $s_{*} \pi_{*}(\bar{y})$ is even.

\section{An application}

Additional assumption Let us assume that the image $\zeta_{*}\left(\left[L^{n-4 k}\right]\right)$ is in the subgroup

$$
\left.\operatorname{Im}\left(I_{1}\right)_{*}+\operatorname{Im}\left(I_{2}\right)_{*} \subset H_{n-4 k}\left(B\left(\left(\mathbb{Z}_{4} \times \mathbb{Z}_{4}\right) \rtimes \mathbb{Z}_{2}\right) \rtimes \mathbb{Z} ; \mathbb{Z}\right)\right) .
$$

Theorem 2. Assume that $n \equiv 3(\bmod 4), k$ is an even number, $n-4 k \geq 7$. Let us assume that the additional condition above is satisfied: Then $\eta_{*}\left(\left[N^{n-2 k}\right] \in H_{n-2 k}(B \mathbb{Z} / 4 \rtimes \mathbb{Z} ; \mathbb{Z})=\mathbb{Z}_{4}\right.$ is an even element.

Proof. Let $\zeta_{*}\left(\left[L^{n-4 k}\right]\right)=x+y$, where $x=\left(I_{1}\right)_{*}(u)$ and $y=\left(I_{2}\right)_{*}(v)$ for suitable elements $u$ mïS $v$. Using the transfer homomorphism, we get: $\bar{\zeta}_{*}\left(\left[\bar{L}^{n-4 k}\right]\right)=\bar{x}+\bar{y}$, where $\bar{x}=\left(\bar{I}_{1}\right)_{*}(\bar{u})$ mïS $\bar{y}=\left(\bar{I}_{2}\right)_{*}(\bar{v})$.

Let us consider the following commutative diagram:

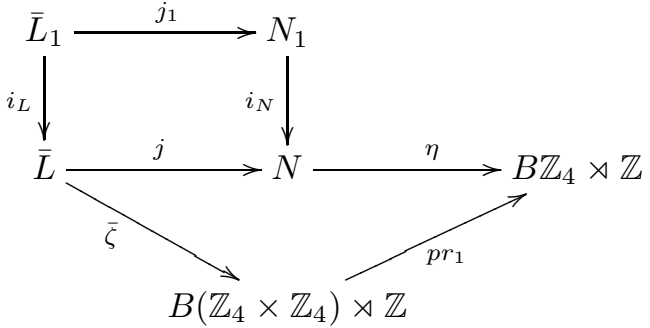

and calculate the image $\left[L_{1}\right] \in H_{n-4 k-4}\left(\bar{L}_{1}, \mathbb{Z}\right)$ in the group $H_{n-4 k-4}\left(B \mathbb{Z}_{4} \rtimes \mathbb{Z}, \mathbb{Z}\right)=\mathbb{Z}_{4}$ by two different way, using the diagram.

At the first step, the image is given by the formula: $\left(\eta \circ i_{N} \circ j_{1}\right)_{*}\left[\bar{L}_{1}\right]$. Let us apply to the both sides of the formula (3) the homomorphism $\eta_{*}$, we get

$$
\left(\eta \circ i_{N} \circ j_{1}\right)_{*}\left[\bar{L}_{1}\right]=(\eta \circ j)_{*}[\bar{L}] \cap e^{2}=\left(p r_{1}\right)_{*}\left(\zeta_{*}[\bar{L}]\right) \cap e^{2}=\left(p r_{1}\right)_{*}(\bar{x}+\bar{y}) \cap e^{2} .
$$

At the second step, let us represent this element as follows: $\left(p r_{1} \circ \bar{\zeta} \circ i_{L}\right)_{*}\left[\bar{L}_{1}\right]$. Using corollaries 1 and 2(a), we get:

$$
\left.\left(p r_{1} \circ \bar{\zeta} \circ i_{L}\right)_{*}\left[\bar{L}_{1}\right]=\left(p r_{1}\right)_{*} \circ(\bar{\zeta})_{*}\left([\bar{L}] \cap\left(\left.\tau^{*}\left(\left.e\right|_{L}\right) \cup e\right|_{L}\right)\right)=\left(p r_{1}\right)_{*}\left(\bar{\zeta}_{*}[\bar{L}] \cap\left(\tau^{(} e_{0}\right) \cup e_{0}\right)\right)=\left(p r_{1}\right)_{*}(-\bar{x}+\bar{y}) \cap e^{2} .
$$

The results of calculations (4) and (5) coincide, then we get $2\left(p r_{1}\right)_{*}(\bar{x}) \cap e^{2}=0$ in the group $H_{n-4 k-4}\left(B \mathbb{Z}_{4} \rtimes\right.$ $\mathbb{Z}, \mathbb{Z})=\mathbb{Z}_{4}$, therefore the element $\left(p r_{1}\right)_{*}(\bar{x}) \cap e^{2}$ is even. By corollary 2 (b) the element $\bar{y}$ is even, this proves that the element $\left(p r_{1}\right)_{*}\left(\zeta_{*}[\bar{L}]\right) \cap e^{2}=\eta_{*}\left(j_{*}[\bar{L}]\right) \cap e^{2}$ is even.

From the Herbert Formula for the immersion $g$ we get: $\eta_{*}\left(j_{*}[\bar{L}]\right) \cap e^{2}=-\eta_{*}([N]) \cap e^{k+2}$. Because the cap-product with the Euler class $-\cap e$ is an isomorphism, the element $\eta_{*}([N])$ is even.

The authors were supported in part by RFBR Grant No 15-01-06302.

\section{References}

[1] Herbert R.J. Multiple points of immersed manifolds. Providence (RI): AMS, 1981 (Mem. AMS; v. 250)

[2] P.J.Eccles, M.Grant Bordism classes represented by miltiple point manifolds of immersed manifolds,

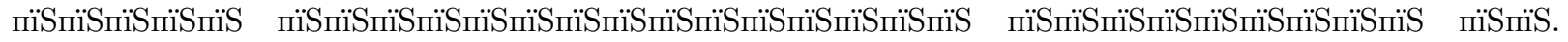

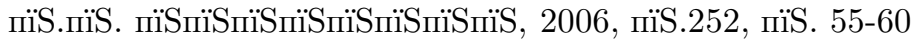




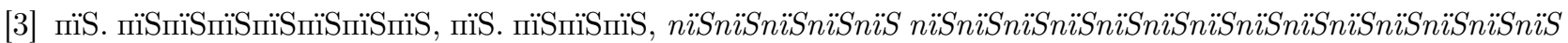
nïSnïSnïSnïSnïSnïSnïS nïSnïSnïSnïSnïSnïSnïSnïS nïSnïSnïSnïSnïSnïSnïS nïSnïSnïSnïSnïS,

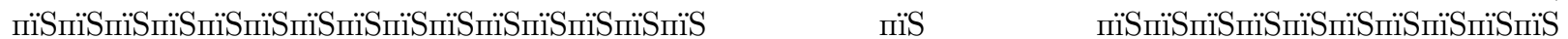

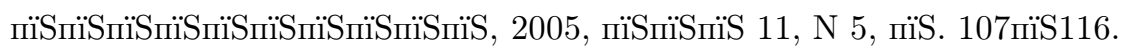

[4] P.M.Akhmet'ev, P.J.Eccles. The relationship between framed bordism and skew-framed bordism // Bull. Lond. Math. Soc. 39, No. 3, 473-481 (2007).

[5] P.Akhmetiev, O.Frolkina. On non-immersibility of $\mathbb{R P}^{10}$ to $\mathbb{R}^{15} / /$ Topology and its Applications, V.160, 11 (2013). 1241-1254.

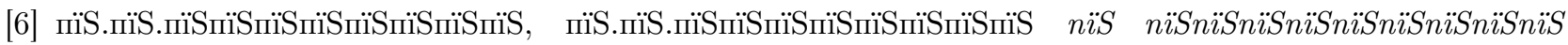

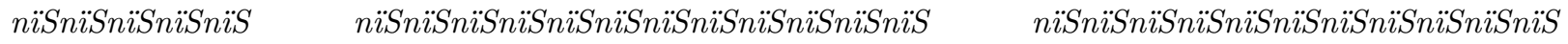

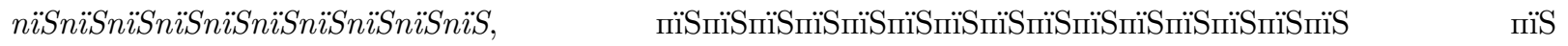

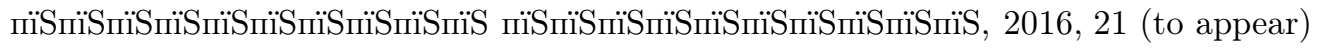

[7] P.J.Eccles. Multiple points of codimension one immersions. Topology Symposium, Siegen 1979, Lecture Notes in Math., vol. 788, Springer, Berlin, 1980, pp. 23пїS38.

[8] M.Grant. Bordism of Immersions. Thesis. 2006.

[9] M.W.Hirsch. Immersions of manifolds. Trans. Amer. Math. Soc. 93 (1959), 242-276.

[10] L.S.Pontryagin. Smooth manifolds and their applications to homotopy theory. Transl., II. Ser., Am. Math. Soc. 11, 1-114 (1959). Translated from: Trudy Mat. Inst. im. Steklov. no. 45. Izdat. Akad. Nauk SSSR, Moscow, 1955.

[11] A.Szücs, Cobordism of immersions and singular maps, loop spaces and multiple points, Geometric and Algebraic Topology, Banach Center Publ., 18 PWN, Warsaw (1986) 239-253.

[12] R.Wells, Cobordism groups of immersions. // Topology 5 (1966) 281- 294. 